Meta

Journal des traducteurs

Translators' Journal

\title{
Variation dénominative et familiarité en tant que source d'incertitude en traduction médicale
}

\author{
Esperanza Alarcón-Navío, Clara Inés López-Rodríguez et Maribel \\ Tercedor-Sánchez
}

Volume 61, numéro 1, mai 2016

Des zones d'incertitudes en traduction

URI : https://id.erudit.org/iderudit/1036986ar

DOI : https://doi.org/10.7202/1036986ar

\section{Aller au sommaire du numéro}

Éditeur(s)

Les Presses de l’Université de Montréal

ISSN

0026-0452 (imprimé)

1492-1421 (numérique)

Découvrir la revue

Citer cet article

Alarcón-Navío, E., López-Rodríguez, C. I. \& Tercedor-Sánchez, M. (2016). Variation dénominative et familiarité en tant que source d'incertitude en traduction médicale. Meta, 61(1), 117-144. https://doi.org/10.7202/1036986ar

\section{Résumé de l'article}

Les traducteurs sont souvent contraints de faire un choix parmi une multitude de variantes terminologiques ayant un signifié similaire. La variation dénominative, également dénommée variation terminologique, est source d'incertitude dans l'esprit du traducteur médical, tant sur le plan cognitif, que sur le plan des équivalences. L'incertitude est présente dès lors que le traducteur ne sait pas si le lecteur d'arrivée sera familiarisé avec la variante choisie dans sa traduction. Les défis soulevés par la variation suffisent à eux seuls à justifier la création d'outils adaptés aux besoins des traducteurs. VariMed est une base de données terminologiques axée sur les motivations cognitives et communicatives de la variation dénominative, et la manière dont elles se reflètent dans les variantes terminologiques d'un concept.

L'objectif de l'article consiste à explorer les zones d'ombre que recèle la traduction de la variation dénominative en traduction médicale sur le plan intralinguistique et interlinguistique dans le cadre des projets de recherche VariMed et CombiMed. Nous aborderons également la perception cognitive de la familiarité (Delgado 1988; Connine, Mullennix et al. 1990 ; Gómez Veiga, Carriedo López et al. 2010) chez les sujets profanes en tant que source complémentaire de doute de la part du traducteur. À cet effet, nous avons mené auprès d'étudiants en traduction une expérience basée sur des tests spontanés de production lexicale afin de connaître leur perception des termes les plus familiers à partir d'éléments extraits de la base de données VariMed. Les résultats de l'expérience ont permis de mieux définir les paramètres qui influencent la familiarité des sujets avec les mots et les termes et, de là, les choix lexicaux des traducteurs.
Ce document est protégé par la loi sur le droit d'auteur. L’utilisation des services d’Érudit (y compris la reproduction) est assujettie à sa politique d'utilisation que vous pouvez consulter en ligne.

https://apropos.erudit.org/fr/usagers/politique-dutilisation/ 


\title{
Variation dénominative et familiarité en tant que source d'incertitude en traduction médicale*
}

\author{
ESPERANZA ALARCÓN-NAVío \\ Universidad de Granada, Grenade, Espagne \\ ealarcon@ugr.es
}

\author{
CLARA INÉS LÓPEZ-RODRÍGUEZ \\ Universidad de Granada, Grenade, Espagne \\ clarailr@ugr.es
}

\author{
MARIBEL TERCEDOR-SÁNCHEZ \\ Universidad de Granada, Grenade, Espagne \\ itercedo@ugr.es
}

\section{RÉSUMÉ}

Les traducteurs sont souvent contraints de faire un choix parmi une multitude de variantes terminologiques ayant un signifié similaire. La variation dénominative, également dénommée variation terminologique, est source d'incertitude dans l'esprit du traducteur médical, tant sur le plan cognitif, que sur le plan des équivalences. L'incertitude est présente dès lors que le traducteur ne sait pas si le lecteur d'arrivée sera familiarisé avec la variante choisie dans sa traduction. Les défis soulevés par la variation suffisent à eux seuls à justifier la création d'outils adaptés aux besoins des traducteurs. VariMed est une base de données terminologiques axée sur les motivations cognitives et communicatives de la variation dénominative, et la manière dont elles se reflètent dans les variantes terminologiques d'un concept.

L'objectif de l'article consiste à explorer les zones d'ombre que recèle la traduction de la variation dénominative en traduction médicale sur le plan intralinguistique et interlinguistique dans le cadre des projets de recherche VariMed et CombiMed. Nous aborderons également la perception cognitive de la familiarité (Delgado 1988; Connine, Mullennix et al. 1990; Gómez Veiga, Carriedo López et al. 2010) chez les sujets profanes en tant que source complémentaire de doute de la part du traducteur. À cet effet, nous avons mené auprès d'étudiants en traduction une expérience basée sur des tests spontanés de production lexicale afin de connaître leur perception des termes les plus familiers à partir d'éléments extraits de la base de données VariMed. Les résultats de l'expérience ont permis de mieux définir les paramètres qui influencent la familiarité des sujets avec les mots et les termes et, de là, les choix lexicaux des traducteurs.

\section{ABSTRACT}

Translators often face the challenge of choosing among a plethora of terminological variants which have a similar meaning. Denominative variation, also known as terminological variation, causes uncertainty in the mind of the translator not only when looking for translation equivalents but also from a cognitive point of view. Uncertainty is also an issue when the translator is not sure whether the target audience will be familiar with the terminological variant $\mathrm{s} / \mathrm{he}$ has chosen. Uncertainty is a real challenge for translators and researchers alike requiring the creation of terminological databases adapted to the needs of translators. VariMed is a terminological resource which focuses on the cognitive and communicative motivations of denominative variation, and their reflection in the terminological variants of a concept. 
The aim of this paper is to explore the grey areas related to the translation of denominative variation in medical translation, a phenomenon taking place both at the intralinguistic and interlinguistic level within the framework of the research projects VariMed et CombiMed. We also study the cognitive phenomenon of familiarity (Delgado 1988; Connine, Mullennix et al. 1990; Gómez Veiga, Carriedo López et al. 2010) among nonexperts as an additional source of doubt on the part of the translator. To this end, we experimentally studied which terminological variants trainee translators perceived as more familiar in spontaneous production tasks based on data from the VariMed database. The results of the experiment shed some light on the parameters that influence familiarity with words and terms, and thus, the lexical choices of translators.

\section{MOTS CLÉS/KEYWORDS}

variation dénominative, variation terminologique, traduction médicale, base de données terminologique, familiarité des termes, VariMed

denominative variation, terminological variation, medical translation, terminological database, term familiarity, VariMed

\section{Introduction : traduction et incertitude}

Le processus de traduction prend comme point de départ un élément concret: le texte et la commande de traduction qui l'accompagne, mais à partir de là émergent de nombreux facteurs qui vont activer certains choix dans le cerveau du traducteur et conditionner le résultat final. Le traducteur doit ainsi faire face à l'incertitude en traduction.

L'incertitude en traduction se manifeste aussi bien sur le plan cognitif (puisqu'elle concerne la compréhension) que sur le plan des équivalences. D’une part, l'élément cognitif intervient dès lors que le traducteur n'est pas sûr des connaissances préalables ni des présuppositions culturelles du destinataire de la traduction. D'autre part, la compréhension des termes se produit au sein d'un réseau de concepts entre lesquels s'établissent des relations. Le traducteur doit aussi identifier les facteurs extratextuels (motif, émetteur, récepteur, intention, moyen de communication, dimension spatiale, dimension temporelle) et intratextuels (sujet, présuppositions, structure textuelle, genre, style, lexique, syntaxe, etc.) définis par la commande de traduction (Nord 2005: 41-42). Enfin, il devra aussi prendre des décisions terminologiques pour trouver l'équivalent ou la variante la mieux adaptée à l'usage dans la langue d'arrivée.

En guise d'exemple, nous pourrions envisager, dans le cadre d'une commande de traduction, de traduire de l'anglais vers l'espagnol un texte médical concernant un accident vasculaire cérébral pour une compagnie d'assurance santé. Il est évident que le traducteur réagira différemment s'il s'adresse à un public spécialisé, semispécialisé, profane, jeune ou âgé. Le moyen de transmission déterminera par ailleurs des décisions liées à la future publication de la traduction sur support papier, sur Internet, dans un DVD multimédia, sur support audio, etc. Il faudra vérifier si des sous-titres sont prévus ou si la traduction sera diffusée en voice-over et envisager les variantes géographiques pour le texte cible (l'espagnol du Mexique, de l'Argentine, de l'Espagne, mais aussi des États-Unis, etc.). Le sujet abordé et le motif de la traduction ne seront pas non plus négligeables pour la sélection lexicale du traducteur puisque les différents facteurs de la commande de traduction vont délimiter les variantes dénominatives les plus appropriées au concept //accident vasculaire cérébral// (stroke, en anglais) comme dans l'exemple suivant: 
TABLEAU 1

Variantes dénominatives pour le concept //accident vasculaire cérébral//

\begin{tabular}{|l|l|}
\hline ANGLAIS & ESPAGNOL \\
\hline $\begin{array}{l}\text { Stroke, intracerebral hemorrhage, brain attack, } \\
\text { cerebrovascular accident, CVA }\end{array}$ & $\begin{array}{l}\text { Ictus, derrame cerebral, accidente } \\
\text { cerebrovascular, apoplejía, ACV, accidente } \\
\text { cerebral vascular }\end{array}$ \\
$\begin{array}{l}\text { ischemic stroke } \\
\text { hemorrhagic stroke }\end{array}$ & $\begin{array}{l}\text { ictus isquémico } \\
\text { ictus hemorrágico }\end{array}$ \\
\hline
\end{tabular}

D’autre part, même si le traducteur est parvenu à trouver des équivalences appropriées du point de vue conceptuel, il se peut que le destinataire de cette traduction médicale soit un profane. Dans ce cas, le traducteur doit se poser la question suivante: le lecteur est-il familiarisé avec les termes médicaux employés? Cette question constitue une nouvelle source d'incertitude puisqu'elle implique d'envisager les présuppositions et les connaissances préalables du destinataire de la traduction et sa familiarité avec les termes choisis par le traducteur.

Le traducteur médical doit ainsi faire face au doute, en dépit du fait qu'en traduction médicale, «les défis les plus importants sont ceux qui ont trait à la véracité, clarté et précision du texte d'origine [...]» (Navarro 2009: 9; notre traduction). À cet effet, il convient donc que le traducteur se pose préalablement les questions suivantes:

- Est-il toujours possible d'être précis en traduction médicale?

- Quelles sont les motivations qui sont à l'origine des différentes variantes terminologiques?

- Quels sont les paramètres pertinents avant d'affronter le choix d'un terme plutôt qu'un autre?

- Est-ce que le souci de précision peut entraver la compréhension d'un concept médical de la part du grand public ou même du traducteur?

Ces questions constituent le point de départ de notre travail et la base sur laquelle reposent les objectifs de cette publication:

a) Aborder la variation dénominative en médecine comme source d'incertitude en traduction et décrire les dimensions et les marqueurs des variantes dans le cadre des projets de recherche VariMed et CombiMed.

b) Souligner l'utilité de la base de données VariMed en tant que ressource multimodale bilingue pour l'étude de la variation dénominative en médecine et de la vulgarisation médicale.

c) Examiner la perception de la familiarité du grand public avec les termes médicaux en s'appuyant sur des expériences menées auprès d'étudiants en traduction.

d) Établir les liens entre les concepts de familiarité, d'incertitude et les choix de traduction.

\section{La variation dénominative en médecine et la familiarité}

La variation dénominative fait référence aux diverses manières de désigner un concept. En médecine, à l'instar d'autres domaines des sciences, les concepts médicaux peuvent se lexicaliser de façon différente selon les facteurs d'adéquation à une situation communicative concrète ou bien compte tenu de la facette ou dimension du concept visé (Tercedor-Sánchez et López-Rodríguez 2012: 252-253). 
La base de données VariMed s'appuie sur la théorie de la «cognition incarnée» (embodied cognition), qui considère que la connaissance et les représentations mentales sont incarnées puisqu'elles sont fondées sur des racines corporelles et des expériences sensorielles (Barsalou 2003; voir aussi Connell, Lynott et al. 2012: 1). Nos expériences corporelles sont présentes dans la langue et les dénominations des concepts. Les dénominations des concepts médicaux varient en fonction de l'expérience perceptuelle concrète qui est à la base de ces concepts (Prieto-Velasco et Tercedor-Sánchez 2014), dans la mesure où notre expérience corporelle et notre interaction avec le monde environnant déterminent notre façon de désigner les concepts.

\subsection{La variation dénominative et ses motivations}

En tant que phénomène lexico-sémantique, la variation dénominative fait référence, d'après Tercedor-Sánchez (2011: 181-182), aux représentations linguistiques partageant avec le terme principal certains traits sémantiques et conceptuels qui s'activent dans certains contextes et situations.

Il faut donc chercher les raisons qui donnent naissance à la variation aussi bien sur le plan cognitif que sur le plan communicatif. Les raisons cognitives (Aijón et Serrano 2010; Fernández Silva 2010) prennent en compte le point de vue que le sujet parlant veut mettre en avant, ainsi que les connaissances et la spécialisation de l'émetteur. Par exemple, la maladie oculaire qui entraîne une diminution de la vision périphérique peut être dénommée visión en túnel, visión en cañón de escopeta, visión tubular, visión tuneliforme en espagnol et vision en tunnel, vision en canon de fusil, vision tubulaire, vision en tunnel en français.

En revanche, les raisons d'ordre communicatif (Cabré 1999; Tercedor-Sánchez et Méndez Cendón 2000; Tercedor-Sánchez 2002; Rogers 2004; Bowker et Hawkins 2006; Freixa 2006) sont, quant à elles, déterminées par la situation communicative et les participants. L'une des approches communicatives les plus influentes pour la variation linguistique est celle de Halliday, McIntosh et Strevens (1964: 77). Ces auteurs distinguent entre la variation qui dépend de l'usage (registre) et la variation liée aux caractéristiques des participants durant les actes de communication (dialectes). Cette perspective a été notamment adoptée au sein des études de traduction par des auteurs tels que Hatim et Mason (1990) ou Baker (1992).

La langue nous permet de comprendre la réalité et les éléments de la connaissance du monde de différentes façons (Wierzbicka 1995) en fonction de nombreux facteurs inhérents au concept et à ses caractéristiques ou à la façon dont l'envisage le sujet parlant. Ainsi, la distinction entre ce que le mot signifie vraiment et la façon dont nous interprétons ce sens par le truchement de l'usage est floue. À cet égard, l'adoption du paradigme cognitif implique que soit remis en cause le point de vue objectiviste qui distingue entre la sémantique et la pragmatique des concepts, la différence entre ce que le mot «signifie en réalité » et la connaissance encyclopédique qu'il nous arrive d'avoir des choses auxquelles se rapportent les mots (Lakoff 1987: 138). Le fait que la sémantique et la pragmatique soient ainsi considérées comme un continuum est la clé permettant de comprendre que les unités lexicales d'un concept ont une pertinence égale, ladite pertinence dépendant d'ailleurs du contexte particulier dans lequel le terme est utilisé. 


\subsection{La familiarité}

Dans les cas où le destinataire d'une traduction médicale est un profane, le traducteur devra se demander si ce lecteur est familiarisé avec les termes médicaux employés.

La perception de la familiarité pour les profanes en médecine est un paramètre intervenant tant dans la communication médecin-patient que dans le choix du traducteur. La familiarité du sujet parlant avec le terme est un des descripteurs cognitifs des variantes. Cette perception de familiarité intervient dans la sélection lexicale et est liée à la compréhension des concepts spécialisés et à la représentation individuelle des connaissances. D’après Delgado Corujo (1988; cité dans Guzmán Rosquete et Jiménez González 2001), on entend par «familiarité», la fréquence subjective avec laquelle un sujet a employé, lu ou entendu un mot. La familiarité représente un élément dont il faut tenir compte que ce soit pour la constitution de ressources terminologiques ou pour la traduction et la rédaction des textes de vulgarisation. La familiarité d'un certain terme pour un sujet (profane ou expert) peut être déterminée selon différents paramètres qui seront abordés dans la section 5 .

La motivation de la sélection d'une dénomination pour un concept est cognitive (multidimensionnalité) ou fonctionnelle (usage géographique, registre, niveau de spécialisation du texte). Il convient de savoir à quel point les patients comprennent la terminologie employée par le personnel en santé et si ces professionnels sont capables d'adapter leur discours aux connaissances médicales des patients, à leur compréhension de la maladie et à la terminologie que le patient emploie pour décrire et désigner la maladie dont il souffre. Par conséquent, l'un des défis de VariMed (Tercedor-Sánchez, López-Rodríguez et Prieto-Velasco 2014) et CombiMed consiste à étudier aussi bien la fréquence objective des unités lexicales au sein d'un corpus de textes que leur fréquence subjective en termes de familiarité. Cette perspective nous permet «to capture characteristics of words as learned by particular individuals, as opposed to characteristics of words as estimated over an extensive language output of a large linguistic community, i.e. a corpus» (Kuperman et Van Dyke 2013).

Grâce aux projets VariMed et CombiMed, il est possible d'étudier la familiarité, ainsi que les phénomènes de compréhension et de production lexicale. Le recueil des données concernant la familiarité est indispensable pour adapter une base de données terminologique à un groupe concret d'usagers. La familiarité dépend de facteurs tels que l'âge d'acquisition et la fréquence objective et subjective d'une unité lexicale (Connine, Mullennix et al. 1990). Guzmán Rosquete et Jiménez González (2001) ont constaté une corrélation entre la fréquence des mots imprimés et la familiarité subjective uniquement dans le cas des mots très fréquents. En fait, l'effet de fréquence lexicale (word frequency effect) intervient dans la langue anglaise avec des mots fréquents et se traduit par une reconnaissance plus rapide des mots communément employés (par exemple, world) face aux mots les moins communs (par exemple, glitch) (Yap, Balota et al. 2012).

Nous partageons l'avis de Gómez Veiga, Carriedo López et al. (2010: 31) et celui de Kuperman et Van Dyke (2013), selon lesquels la fréquence subjective peut être considérée comme une variable plus utile que la fréquence de corpus lorsqu'il s'agit d'études menées auprès de populations d'enfants ayant un faible taux d'alphabétisation. En outre, la fréquence subjective est considérée en tant que variable essentielle dans la compréhension des textes (Alderson 2007) et certains éléments portent à 
croire que la fréquence subjective est plus fiable que la fréquence des mots dans un corpus textuel. En fait, il existe un rapport entre la fréquence subjective et l'accès lexical du sujet au lexicon mental.

D'autres recherches permettent d'affirmer que les experts ont tendance à sousestimer les connaissances des profanes (Bromme, Rambow et al. 2001). Dans le but de mieux évaluer le degré de familiarité des sujets profanes avec certains concepts médicaux et leurs lexicalisations, nous avons mené une étude visant à établir la correspondance entre les définitions et les différentes options lexicales véhiculées par les concepts auxquels celles-ci font référence. Notre expérience a pour objectif, au moyen des jugements de mémoire de reconnaissance (recognition memory judgements) (Mandler 1980; Jacoby, Yonelinas et al. 1997), d'évaluer l'expérience préalable des sujets soumis à un stimulus (Yonelinas 2002: 445). Cependant, il importe de souligner que la familiarité n'affecte pas seulement la fluidité de la reconnaissance perceptuelle de l'unité lexicale, mais aussi la fluidité conceptuelle; autrement dit, la familiarité améliore la compréhension du signifié de l'unité lexicale de la part du sujet (Yonelinas 2002). Il en résulte que la familiarité est liée aussi bien à la reconnaissance perceptuelle qu'au traitement conceptuel (Jacoby 1991; Jacoby et Kelley 1992), ce qui fait que le sujet a la sensation de «reconnaître quelque chose», même s'il ne sait pas vraiment ce que cela veut dire.

\section{Méthodologie : recherches et expériences sur la variation et la familiarité}

La variation devient ainsi l'un des éléments majeurs du choix du traducteur médical. La variation dénominative a été le sujet du projet de recherche «VariMed: Variation dénominative en médecine», et de son développement ultérieur, le projet «CombiMed: Lexique combinatoire en médecine: cognition, texte et contexte».

Les objectifs principaux en matière de recherche sont, d'une part, l'étude des motivations cognitives, communicatives et culturelles de la sélection lexicale et, de l'autre, l'analyse de la variation dénominative par rapport aux processus de compréhension et de production contextuels. Dans les projets VariMed et CombiMed, l'étude de la compréhension des variantes a été développée à partir des expériences sur les métaphores et la familiarité, tandis que les termes analysés ont été extraits de corpus parallèles et comparables en espagnol et en anglais. À ces travaux s'ajoutent également des études expérimentales menées avec des étudiants de traduction dans ce domaine de production de variantes (Tercedor-Sánchez, López-Rodríguez et Alarcon 2013).

Les motivations cognitives et communicatives de la variation dénominative déclenchent non seulement des mécanismes de création lexicale en néologie, mais peuvent aussi fournir des explications sur la sélection lexicale que font les sujets parlants d'une langue quand ils communiquent. Ainsi, dans la communication médecin-patient, les descriptions issues des symptômes de la part du patient sont à l'origine de variantes dénominatives fondées sur une métaphore, telles que dans le type de vision caractéristique du glaucome, dénommée moscas volantes [mouches volantes]. Ici, la métaphore a été préférée au terme spécialisé miodesopsia [myodésopsie]. Par ailleurs, pour des raisons communicatives, les spécialistes désignent la maladie à l'aide de termes euphémiques: degeneración macular asociada a la edad [dégénérescence maculaire liée à l’âge], au lieu de degeneración macular senil [dégé- 
nérescence maculaire sénile], síndrome bipolar [syndrome bipolaire], au lieu de síndrome maníaco depresivo [syndrome maniaco-dépressif]. Ces euphémismes peuvent entraîner un changement de la perception de familiarité de la part du patient.

La présente section permettra d'aborder les applications possibles de la base de données VariMed pour l'étude de la variation dans le cadre des projets VariMed et CombiMed et les différents paramètres susceptibles d'expliquer les motivations de la variation et de la familiarité. Nous présenterons et analyserons ensuite les résultats d'une expérience consacrée à l'étude de la familiarité auprès d'étudiants de traduction spécialisée, ce qui nous permettra d'établir des rapports entre variation dénominative, choix de traduction, familiarité et incertitude en traduction.

\subsection{L'étude de la variation: la base de données VariMed}

La base de données VariMed a pour objet d'étude la variation médicale. Sa structure repose sur deux grands piliers, à savoir le niveau intralinguistique en espagnol (acné - granos - espinillas - barros - granitos; diarrea del turista - diarrea del viajero venganza de Moctezuma) et en anglais (Moctezuma's revenge - traveler's diarrhea) et le niveau interlinguistique anglais-espagnol (keyhole surgery / laparoscopia; constipation / estreñimiento). La variation géographique constitue une manifestation importante de la variation intralinguistique à prendre en compte. En guise d'exemple, nous pouvons citer le concept //tos ferina// [coqueluche], qui adopte les formes lexicales suivantes en espagnol: tos ferina, tosferina, tos convulsiva et la variante hispano-américaine coqueluche, qui est d'ailleurs un emprunt du français.

VariMed constitue un outil multifonctionnel s'adressant à différents usagers, allant du grand public aux traducteurs, en passant par les médecins et autres professionnels de la santé ou même par les linguistes. L'exploitation de cette base de données peut donc viser aussi bien la vulgarisation médicale ou la recherche linguistique, que la rédaction de textes ou la traduction proprement dite. L'outil de consultation permettant des recherches de base et personnalisées, l'exploitation des variantes de VariMed offre aussi des possibilités d'application en didactique de la traduction.

\subsubsection{Recherches dans VariMed}

VariMed est un outil polyvalent qui permet de faire des recherches de base, soit en ciblant les concepts ou variantes, soit en visant directement les images. Ainsi, à partir d'une recherche telle que visión en túnel (voir Figure 1), on peut consulter ses variantes et avoir accès à d'autres paramètres. 


\section{FIGURE 1}

Recherche du concept/variante visión en túnel dans VariMed

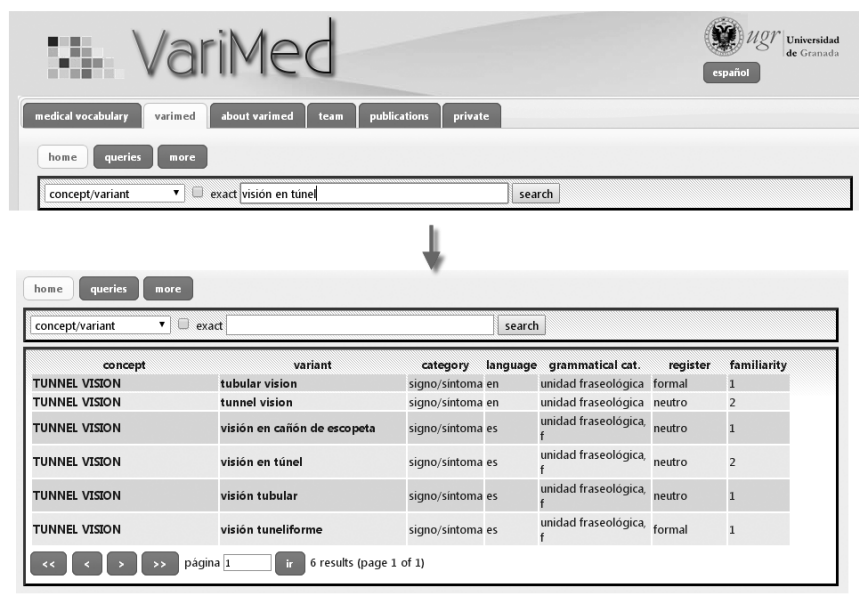

Voici ci-dessous, la fiche du concept //visión en túnel - tunnel vision// (Figure 2). Elle contient une définition, des traits conceptuels (type de concept, concepts associés, partie du corps associée), une image, ses variantes principales en espagnol et en anglais, et des informations administratives (auteur de la fiche, date de création et de révision).

\section{FIGURE 2}

Recherche du concept //visión en túnel// dans VariMed

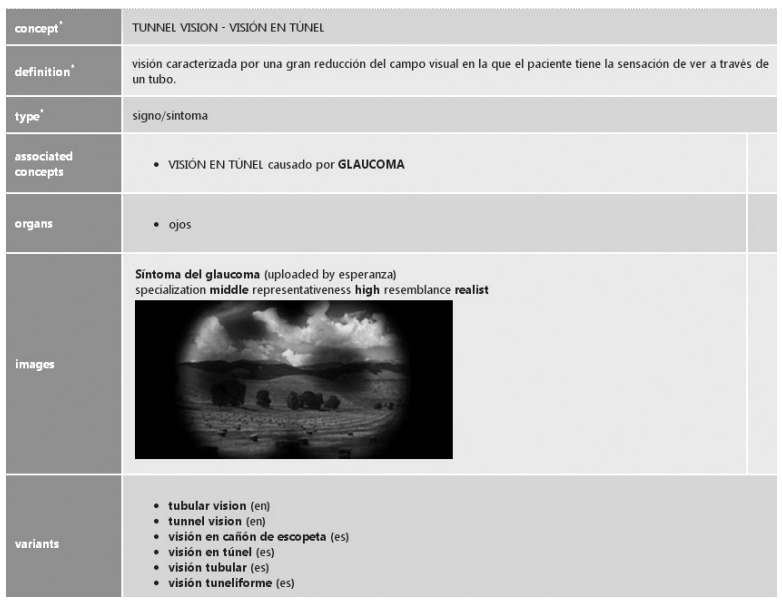

Il est également possible d'approfondir la recherche concernant certaines variantes, en l'occurrence, visión en cañón de escopeta (Figure 3). La fiche de chaque variante permet aussi d'afficher différentes dimensions établies sur un plan cognitif (par exemple, métaphore, attribut visuel, concept, degré de familiarité) comme sur un plan communicatif et culturel (registre, traits géographiques, temporels et sociaux). 
Figure 3

Recherche de la variante visión en cañón de escopeta dans VariMed

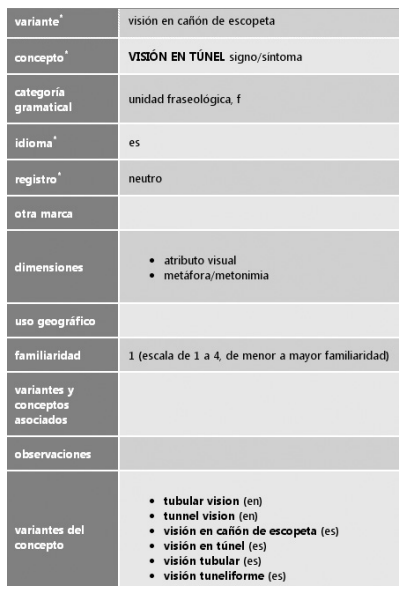

Il est par ailleurs possible d'entreprendre des recherches personnalisées. Ce type de recherche permet de cibler certaines dimensions et, par là, d'étudier les concepts d'après leur type (maladie, signe/symptôme), les concepts qui y sont associés, la partie du corps concernée, les images associées et leur degré de représentativité, de spécialisation et de ressemblance.

À partir de variantes, cette modalité de recherche donne la possibilité d'identifier les concepts ou variantes associés, les variantes qui privilégient une certaine motivation cognitive (métaphore, la relation agent/résultat, etc.), la langue (anglais ou espagnol), la catégorie grammaticale, le registre ou les traits géographiques, temporels et sociaux de cette variante. VariMed a également incorporé les contextes des variantes, ainsi que les coordonnées des sources citées (référence bibliographique, ressources en ligne, etc.). L'exemple de la Figure 4 illustre comment retrouver les variantes relatives au terme espagnol visión et faire une recherche sur les métaphores associées aux multiples formations lexicales créées à partir du concept ou variante visión.

\section{FIGURE 4}

\section{Recherche du concept/variante visión dans VariMed}

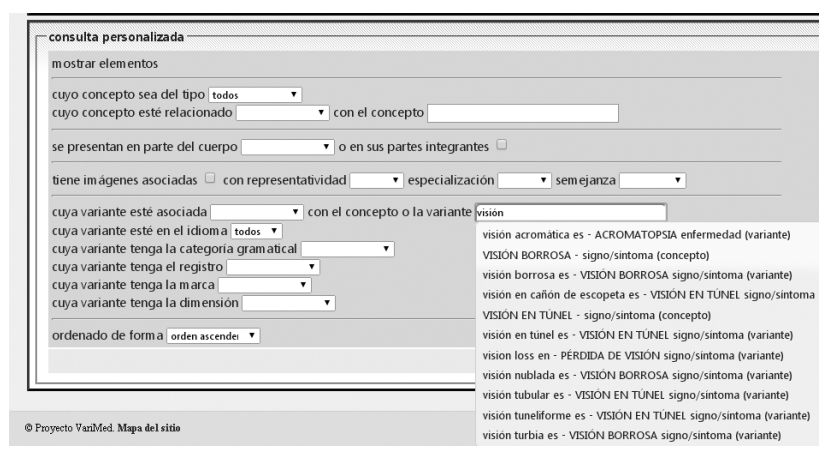

Ainsi, différents descripteurs sont attribués aux variantes recensées, le but étant que les consultations puissent s'adapter aux besoins des usagers et que la composante cognitive des concepts soit ainsi mise en évidence. 


\subsubsection{Représentation des motivations cognitives et communicatives dans VariMed}

Les différentes dimensions d'ordre cognitif retenues dans le domaine des variantes des termes médicaux sont les suivantes: l'origine géographique, la population concernée, la relation agent/résultat, l'attribut visuel ou non visuel, la référence aux composantes de certains concepts, la phase temporelle de la maladie, le degré d'intensité, l'identité du chercheur-découvreur et la localisation de la maladie.

Dans l'exemple qui suit (Figure 5), le choix visión en cañón de escopeta est motivé par le souhait ou le besoin d'employer ce terme métaphorique plutôt que des variantes telles que visión tubular, visión en túnel ou visión tuneliforme, en fonction des connaissances de l'auteur du texte, du genre textuel et des caractéristiques du destinataire de la traduction.

\section{FIGURE 5}

Interface permettant d'introduire les dimensions cognitives concernées par une variante de VariMed

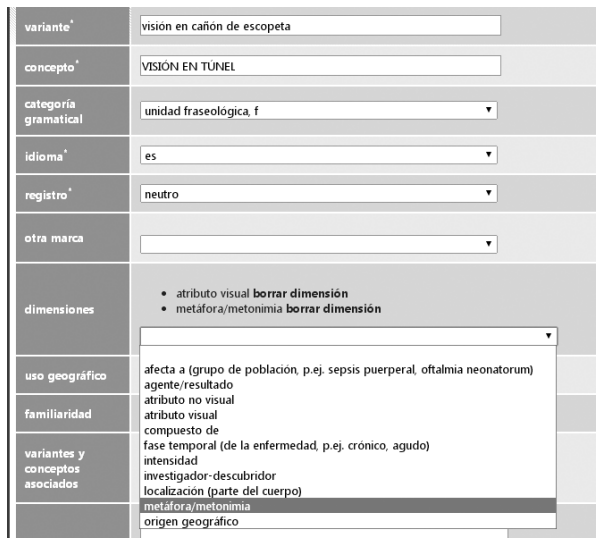

Outre la motivation cognitive, la sélection terminologique dépend également de facteurs communicatifs et situationnels qui permettent de caractériser une certaine communauté linguistique. Sur ce plan, la variation dépend de l'usage et de l'usager. C'est là qu'entrent en jeu le registre (formel, informel, expert, etc.) et les traits géographiques (anglais américain, espagnol européen, etc.), temporels et sociaux. Les marqueurs concernant l'usage (Figure 6), quant à eux, mettent l'accent sur : les abréviations,

\section{FIGURE 6}

\section{Interface de VariMed indiquant les marqueurs d'usage}

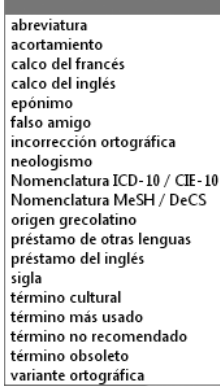


les troncations, les calques du français ou de l'anglais, les éponymes, les faux amis, les néologismes, les nomenclatures, l'origine gréco-latine, les emprunts de l'anglais, les sigles, les termes culturels, les termes les plus employés, les termes à ne pas employer, les erreurs orthographiques, les termes désuets et les variantes orthographiques.

En définitive, il s'agit d'inventorier les variantes terminologiques dans les langues concernées (l'espagnol et l'anglais dans le cas de VariMed) et de les caractériser sur le plan sémantique et pragmatique en allant au-delà des schémas basiques que sont le terme, la définition, le contexte (référence) et l'équivalence, pour aborder la situation communicative et l'usage des termes. Ce qui nous amènera à passer de la présentation de la base à celle d'une expérimentation.

\subsection{Plan expérimental: questions de recherche, sujets, questionnaire et stimuli}

Dans la recherche des concepts médicaux, il est important de distinguer entre les concepts que le médecin est seul à connaître de manière prototypique grâce à sa maîtrise du domaine spécialisé et ceux qui font partie de la connaissance du monde de la part du profane. En s'appuyant sur cette différence initiale, la base de données VariMed établit notamment une distinction entre les marques de registre (formel, informel, enfants, neutre), la présence de textes de vulgarisation générale par opposition aux textes de niveau expert-expert et la représentation de ces concepts au sein d'un système notionnel (les concepts les plus haut placés dans une hiérarchie sont a priori mieux connus du grand public que ceux qui occupent des niveaux inférieurs). En tant que terminographes, notre recherche visait à déterminer si les sujets choisissaient comme terme le plus familier celui qui avait été introduit dans la base de données VariMed pour dénommer et étiqueter le concept. C’est la raison pour laquelle nous avons conçu une expérience ayant pour but de répondre aux questions qui suivent.

\subsubsection{Questions de recherche}

Quels facteurs peuvent influencer le choix d'un terme comme étant le plus familier?

Quelle serait l'influence de la langue maternelle et du transfert entre langues dans la familiarité?

Est-il possible d'établir un lien entre choix de traduction, incertitude et familiarité?

\subsubsection{Population étudiée dans le cadre de l'expérience}

L'expérience s'appuie sur un test soumis à un échantillon de 100 étudiants ayant des compétences traductologiques poussées; elle a été réalisée dans le cadre de la matière à option de troisième et quatrième année de Traduction spécialisée de la licence en Traduction et Interprétation de l'Université de Grenade (Espagne). Parmi ces 100 sujets, 50 avaient l'espagnol pour langue maternelle et 50 le français. Les participants étaient âgés de 19 à 27 ans (moyenne recensée de 20 ans et écart type de 1414, dont 6 hommes et 44 femmes) pour ce qui est du groupe d'individus ayant l'espagnol comme L1 et de 19 à 26 ans (moyenne de 20,8 ans et écart type de 1212 dont 4 hommes et 46 femmes) dans le cas des sujets francophones (la plupart d'entre eux étant des étudiants Erasmus d'origine belge et française). 


\subsubsection{Questionnaire et stimuli}

Les sujets ont répondu de façon anonyme et sur la base du volontariat à une épreuve de décision lexicale visant l'association entre un terme et sa définition; ce test a été effectué par les étudiants dans le cadre d'un cours de traduction. Les individus ont reçu des consignes orales et écrites.

En l'occurrence, le test leur demandait: «Señale con una cruz el término que le resulte más familiar en relación con la definición aportada» [Marquez d'une croix le terme se rapportant à la définition correspondante que vous considérez comme le plus familier]. Le choix de cette consigne visait à établir le degré de familiarité des sujets profanes avec les variantes dénotatives d'un concept médical à usage général. Les participants ont reçu des instructions orales complémentaires leur demandant de choisir la première option «qui leur venait à l'esprit» et de ne pas revenir sur leur choix une fois la décision prise, c'est-à-dire de ne pas corriger leur réponse. En effet, les études neurolinguistiques signalent que la familiarité se trouve réduite immédiatement après l'accès lexical - sélection qui se produit au moment de l'activation lexico-cognitive du lien graphème-sens - (Atkinson et Juola 1973) parce que l'incertitude apparaît quand le sujet commence à réfléchir et à essayer d'interpréter l'unité lexicale.

Afin de garantir la «validité écologique» (Saldanha et O’Brien 2014: 33) de l'expérience, c'est-à-dire que la situation expérimentale créée artificiellement puisse être comparée avec les conditions réelles des individus de la population à l'étude, l'épreuve comprenait 30 stimuli choisis en espagnol dans la base de données VariMed. Les items consistaient en des définitions pour des maladies, des sigles et des symptômes communs. Plus précisément, le choix de ces stimuli a été fait sur un total de 1141 concepts en se basant sur leur usage dans des textes de vulgarisation adressés au grand public. Nous présupposons en effet que les sujets non experts vont les identifier et, dans une certaine mesure, les connaître. Les stimuli comportaient des définitions dans lesquelles étaient au moins présentes deux des relations conceptuelles suivantes: [TYPE-OF], [PRODUCED BY], [CAUSE OF], [SITUATED IN]. À chaque définition étaient associées au choix de trois à huit lexicalisations possibles, dont un élément distracteur. Morphologiquement, les lexicalisations se composaient d'unités monolexicales (ictus), polylexicales (accidente cerebral vascular) ou de sigles ( $\underline{\mathrm{ACV}}$ ). Parmi les stimuli, on pouvait distinguer des concepts relatifs: a) aux maladies dont chacun a souffert au cours de sa vie (par exemple, diarrhée); b) aux maladies moins fréquentes mais que les médias diffusent à cause de leur gravité ou de leur prévalence (cancer du sein); et c) aux maladies peu communes.

Tous les stimuli du questionnaire et les résultats sont publiés en annexe à la fin de cet article. L'exemple ci-dessous permet d'observer un cas de stimulus:

Enfermedad infecciosa contagiosa que afecta habitualmente a los niños, causada por un paramixovirus y localizada fundamentalmente en una o ambas glándulas parótidas. [Maladie infectieuse de type contagieux qui touche d'ordinaire les enfants; elle est causée par un paramixovirus et principalement localisée dans l'une ou les deux glandes parotides.]

paperas [oreillons]

parotiditis [parotidite]

paramixotis [ ${ }^{*}$ paramixote, terme distracteur]

parotiditis epidémica [parotidite épidémique] 


\section{Résultats de l'expérience et paramètres de familiarité dérivés}

\subsection{Résultats de l'expérience}

Les résultats de l'expérience montrent que la familiarité est un concept variable qui dépend du sujet et, en grande mesure, de la fréquence des stimuli lexicaux employés (voir Annexe). Par exemple, les termes désignant les maladies les plus courantes (diarrea/diarrhée, resfriado/rhume, gripe/grippe, estreñimiento/constipation, acné/ acné, alergia/allergie), sont souvent les plus familiers et le choix du terme le plus familier coule de source dans ce cas (voir Figures 7 et 8).

\section{FIGURE 7}

Familiarité avec les lexicalisations pour la définition: Afección cutánea caracterizada por un taponamiento de las glándulas sebáceas o los folículos pilosos que puede provocar inflamación e infección. [Affection cutanée due à une obstruction des glandes sébacées ou des follicules pileux pouvant entraîner une inflammation avec infection.]

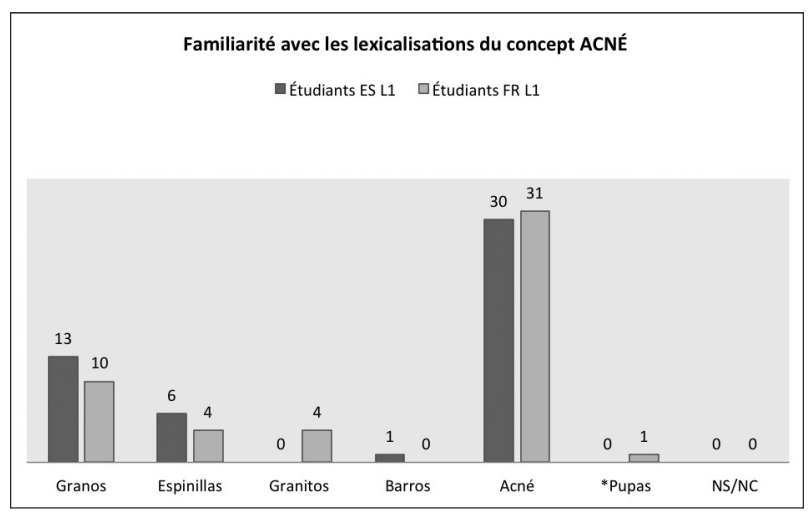

${ }^{*}$ Terme distracteur

\section{FIGURE 8}

Familiarité avec les lexicalisations pour la définition: Proceso infeccioso de las vías respiratorias altas, esencialmente de las fosas nasales, de breve duración (de 5 a 7 días), y de naturaleza benigna, muy común en los meses de invierno en la población general. Es muy contagioso. [Processus infectieux des voies respiratoires supérieures qui atteint principalement les fosses nasales. Il est de courte durée (5-7 jours) et de nature bénigne et très fréquent pendant les mois d'hiver dans la population générale.]

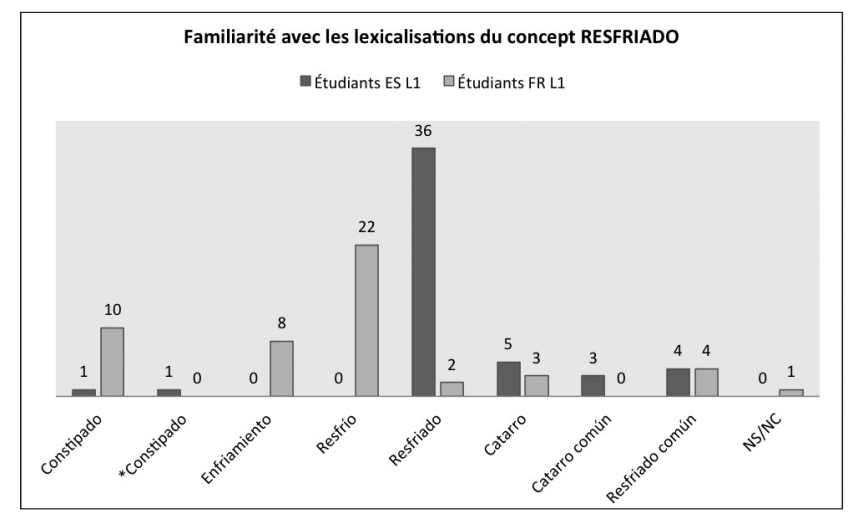

*Terme distracteur 


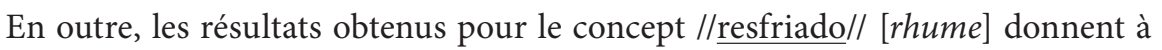
penser qu'il existe une corrélation entre le nombre de choix proposés et le degré d'incertitude dans la détermination du terme le plus familier.

Il est aussi intéressant de s'interroger sur la relation entre, d'une part, la fréquence objective d'une variante dénominative au sein d'un corpus de langue générale et, de l'autre, la familiarité. Comme on le voit dans la Figure 9 ci-dessous, en comparant les résultats des variantes du concept //resfriado// dans le Corpus de référence de l'espagnol actuel (Corpus de Referencia del Español Actual) de l'Académie royale de la langue espagnole (Real Academia Española) et dans l'EsTenTen European ${ }^{1}$, un rapport pourrait être établi entre les termes plus familiers pour les natifs de langue espagnole (resfriado, catarro et enfriamiento) et les termes plus fréquents dans lesdits corpus (resfriado, catarro et enfriamiento). Il apparaît que, d'après les deux corpus consultés, le terme le plus fréquent en langue espagnole est le même que celui que les sujets désignent comme étant le plus familier. Cela apparaît même quand le concept est représenté par diverses variantes dénominatives et que la distribution des résultats entre de multiples options les rend plus équilibrés. Ces résultats confirment, en outre, une expérience préalable qui comparait les variantes choisies par les étudiants dans une épreuve de traduction et leur fréquence dans un corpus (Tercedor-Sánchez, López-Rodríguez et Alarcón-Navío 2013). Il est à noter, toutefois, que certaines études produisent au contraire des résultats montrant une plus grande familiarité avec les mots les moins fréquents (par exemple, Gregg 1976; Glanzer et Adams 1985).

FIGURE 9

Fréquence objective des variantes dénominatives du concept //resfriado// dans des corpus espagnols de langue générale

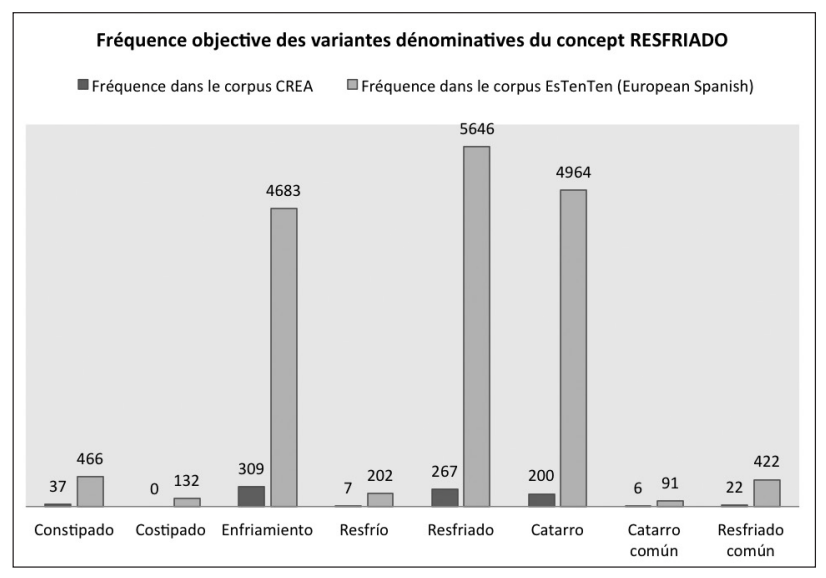

Le fait que les étudiants de français L1 aient choisi comme termes les plus familiers resfrío (élément distracteur), constipado et enfriamiento, qui ont, par contre, très peu retenu l'attention des étudiants espagnols, confirme l'influence des langues maternelles (voir Figure 8). De manière prévisible, les individus francophones montrent davantage de doute dans le choix lexical de termes médicaux en espagnol que ceux qui ont l'espagnol comme L1. Par conséquent, le terme le plus familier pour un hispanophone n'est pas forcément le même pour un francophone (voir Figure 10). 


\section{FIGURE 10}

Familiarité avec les lexicalisations pour la définition: Afección cerebrovascular súbita producida por una falta de riego sanguíneo en una zona del cerebro, debida a la rotura de un vaso sanguíneo o a un coágulo. Se caracteriza por una pérdida súbita y sin convulsiones de la función neurológica. [Affection cérébro-vasculaire subite produite par un manque d'irrigation sanguine dans une zone du cerveau; elle est due à la rupture d'un vaisseau sanguin ou à un caillot. Elle est caractérisée par une perte subite et sans convulsions des fonctions neurologiques.]

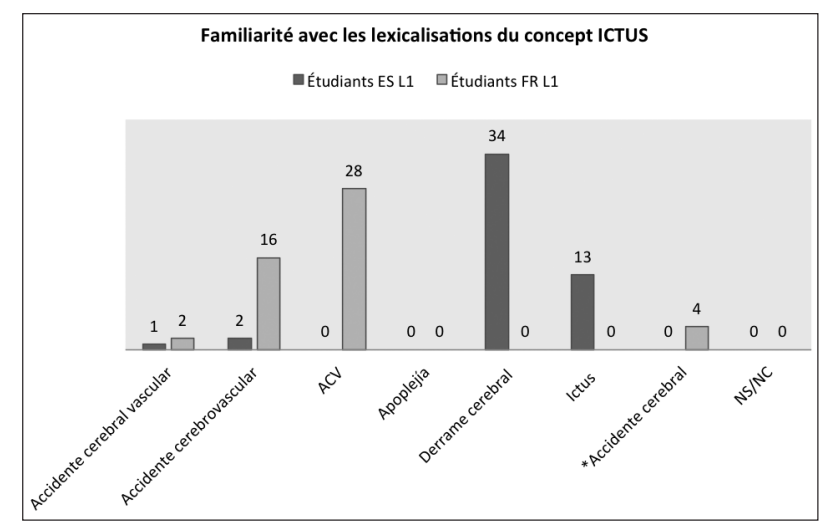

*Terme distracteur

Les sujets francophones laissent apparaître davantage d'incertitude dans la sélection lexicale de termes médicaux en espagnol que ceux qui ont l'espagnol comme $\mathrm{L} 1$, les réponses des participants francophones étant plus variées que celles des Espagnols.

De plus, ils sont plus susceptibles de choisir le mauvais terme (ou élément distracteur) et le terme le moins familier puisqu'ils sont guidés dans leur sélection par d'autres facteurs personnels, extralinguistiques, lexicaux et textuels.

Dans l'exemple d' "ictus" (Figure 10 ci-dessus), 4 sujets ayant le français comme langue maternelle ont choisi l'élément distracteur accidente cerebral. Il faut signaler que contrairement aux individus espagnols qui ont tendance à ne pas choisir les sigles (FR, $\underline{\mathrm{ACV}}$ ) en tant que terme le plus familier, les étudiants francophones ont porté leur choix sur ACV, probablement sous l'influence du sigle français AVC.

Pour les mêmes raisons morphologiques, les participants francophones préfèrent les variantes qui ressemblent au terme le plus familier en français. Nous avons relevé des exemples tels que: estrabismo/strabisme, constipación/constipation, fiebre del

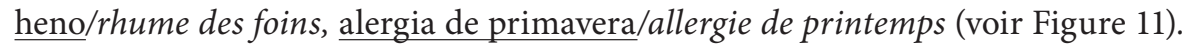




\section{FIGURE 11}

Familiarité avec les lexicalisations du concept //fiebre del heno//: Tipo de alergia estacional que se produce en primavera causada por el polen. [Type d'allergie saisonnière qui survient au printemps en réaction au pollen.]

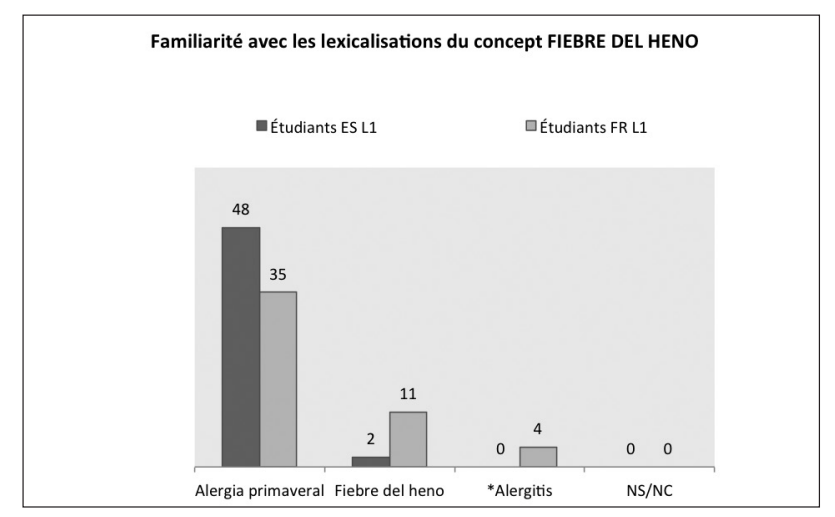

On observe également que le choix de l'hyperonyme par les étudiants francophones (par ex., l'option infección vaginal pour le concept //candidiasis vaginal// [candidose vaginale]) peut être considéré comme une stratégie cognitive pour ne pas prendre de risque et tenter de contourner ainsi l'erreur possible (Figure 12).

\section{FIGURE 12}

Familiarité avec les lexicalisations pour la définition: Infección por cándida localizada en la vagina. [Infection vaginale par Candida.]

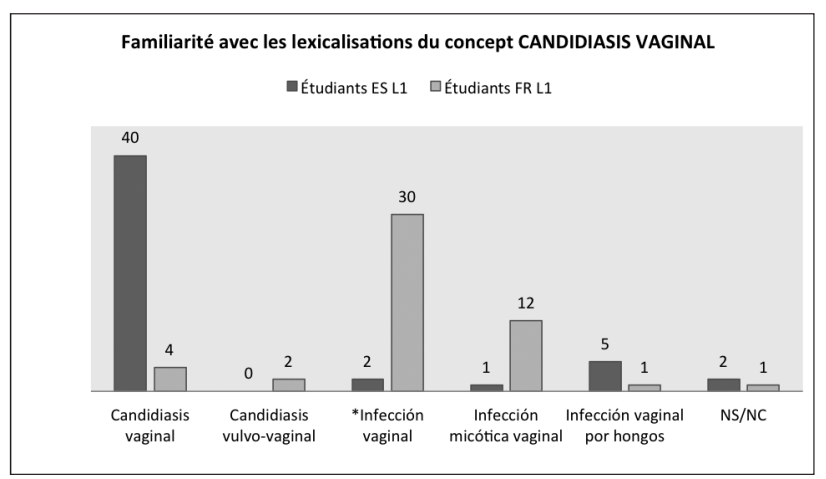

${ }^{*}$ Terme distracteur

En fait, la stratégie consistant à fournir le maximum d'information tout en exigeant le moindre effort cognitif - qui vise à réduire les différences entre les stimuli pour les ramener à des proportions gérables du point de vue cognitif et comportemental - est cohérente avec la sélection du terme considéré en tant que «basic level» (Rosch 1978). Selon l'auteur, «the most basic level of categorization will be the most inclusive (abstract) level at which the categories can mirror the structure of attributes perceived in the world» (Rosch 1978: 30).

En outre, il faut souligner que les termes à racine gréco-latine sont généralement moins familiers dans le groupe de sujets consulté: tonsilitis, influenza, parotiditis, 


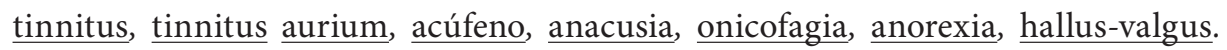
Cependant, les étudiants ont également choisi ictus ou alopecia.

On observe de surcroît que le lecteur ordinaire a davantage de difficultés à établir un lien entre le concept non familier et les termes dont la motivation n'est pas évidente. C'est le cas de conjuntivitis palpebral. Ce terme distracteur a été créé parce qu'il ne permet pas d'associer les variantes métaphoriques leñosa ou lígnea [ligneuse] avec la description de «lesiones pseudomembranosas» présente dans la définition, tandis que la localisation de la lésion (la paupière) est plus familière, notamment pour les francophones (voir Figure 13).

\section{FIGURE 13}

Familiarité avec les lexicalisations pour la définition : Forma rara de conjuntivitis crónica caracterizada por la formación recurrente de lesiones pseudomembranosas, generalmente a nivel de la superficie palpebral. [Forme rare de conjonctivite chronique caractérisée par la formation récurrente de lésions pseudo-membraneuses, le plus souvent au niveau des surfaces palpébrales.]

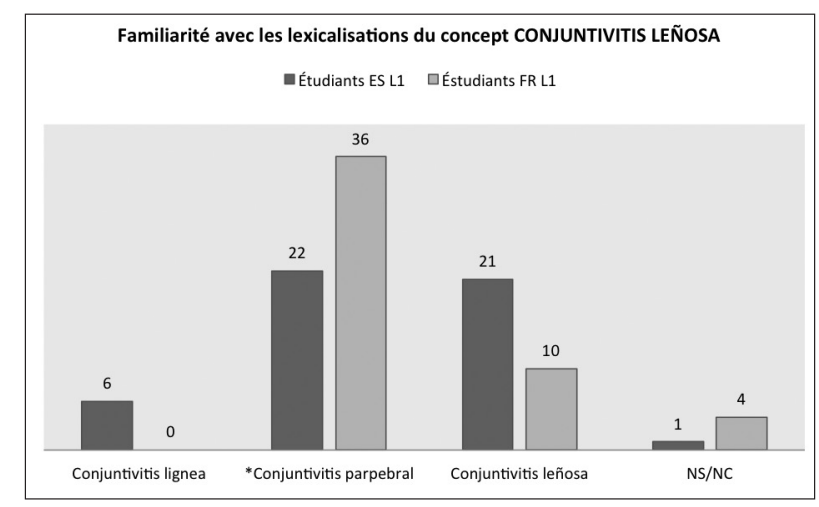

*Terme distracteur

Enfin, la variante la plus familière pour les hispanophones est perçue comme la plus adéquate quand il s'agit, par exemple, d’adapter un texte à un public général ayant des connaissances médicales limitées.

\section{Proposition de paramètres de familiarité}

Les études empiriques issues des projets VariMed et CombiMed (Prieto-Velasco et Tercedor-Sánchez 2014; Tercedor-Sánchez et López-Rodríguez 2012; TercedorSánchez, López-Rodríguez et Alarcon-Navío 2013; Tercedor-Sánchez, López-Rodríguez et Prieto-Velasco 2014) ont permis de jeter les bases de futures études concernant la variation dénominative et la familiarité. Bien qu'il reste à entreprendre des recherches complémentaires sur la variation lexicale, les résultats des études menées dans le cadre des deux projets et l'analyse des données recueillies dans l'expérience décrite ci-dessus nous ont permis de délimiter une série de paramètres qui peuvent favoriser la sélection d'un terme comme étant le plus familier (Figure 14). Ces paramètres seront explorés dans des études ultérieures. 
Figure 14

Proposition de paramètres de familiarité d'après les résultats expérimentaux

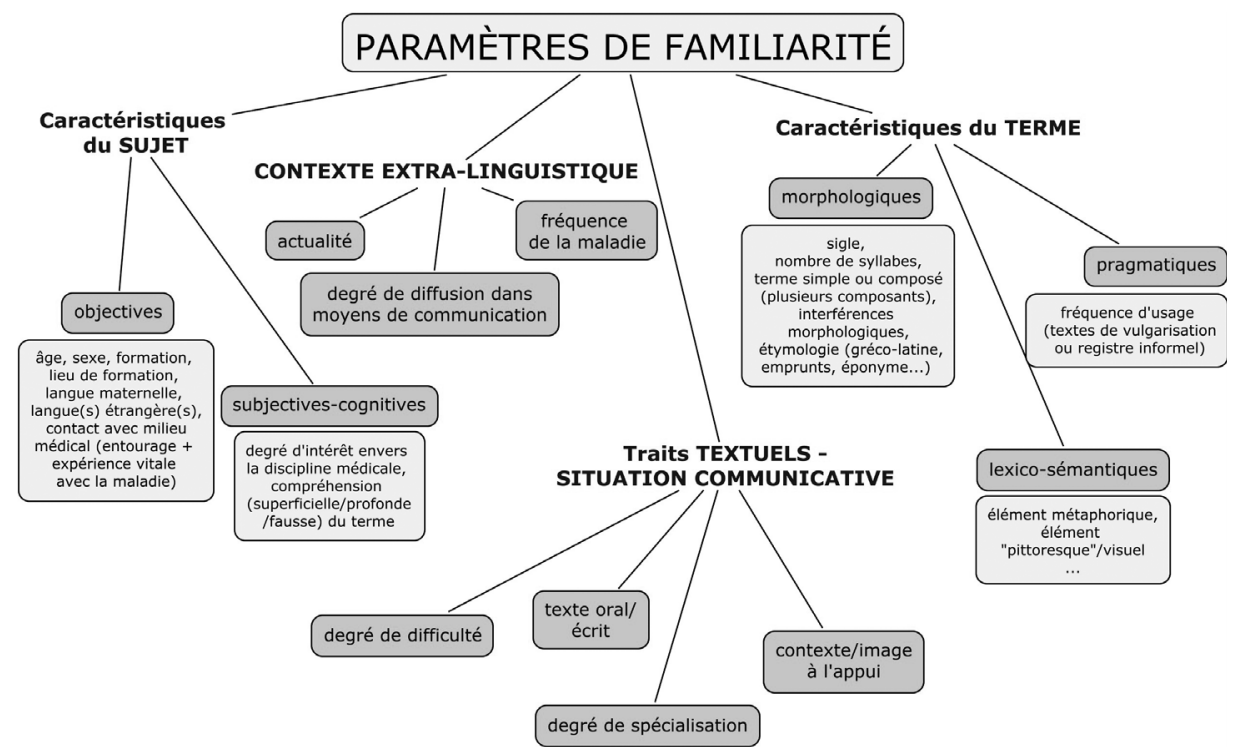

La notion de familiarité nous mène ainsi à distinguer quatre grands axes de paramètres, qui sont autant de sources d'incertitude liées au choix des variantes dénominatives: caractéristiques du sujet, contexte extralinguistique, caractéristiques du terme et du texte dans le cadre d'une situation communicative concrète.

Les caractéristiques du sujet constituent un élément essentiel de la familiarité. On peut distinguer les caractéristiques objectives, telles que l'âge, le sexe, la formation, le lieu de formation, la langue maternelle, la ou les langues étrangères, et le contact avec le milieu médical, tant grâce à l'entourage personnel, l'expérience personnelle de la maladie qu'à l'individu. D'après notre expérience, la langue maternelle (espagnole ou française), la formation universitaire et le lieu de formation (Espagne ou France) ont influencé non seulement la perception de familiarité de la part des sujets, mais aussi la sélection des termes.

Les traits objectifs sont accompagnés de facteurs d'ordre subjectif et cognitif. Sur ce plan, il faudrait tenir compte du degré d'intérêt pour la discipline médicale, ainsi que du niveau de compréhension superficielle, profonde ou fausse du terme.

Le contexte extralinguistique joue également un rôle dans la perception de la familiarité si l'on considère que le sujet reçoit des informations portant sur certaines maladies plus fréquentes (diarrhée, rhume, grippe, acné, etc.) ou qui suscitent une alarme sociale souvent déclenchée par les médias (par exemple, la maladie de la vache folle, la maladie à virus Ebola, la grippe $\mathrm{A}$ [H1N1], etc.) dans un contexte d'actualité ce qui déclenche chez lui un intérêt. Notre expérience a confirmé que la familiarité avec les termes médicaux est liée à la prévalence des maladies.

De même, les caractéristiques morphologiques, pragmatiques et lexico-sémantiques du terme affectent le degré de familiarité ressenti. En effet, la formation simple ou composée du terme, le fait qu'il s'agisse d'un sigle, le nombre de syllabes, l'étymologie du terme (origine gréco-latine, emprunt, éponyme, etc.) représentent autant 
d'éléments liés à la familiarité comme on le voit dans les exemples cités ci-dessus (ACV, tinnitus aurium, influenza, etc.). Sur le plan lexico-sémantique viennent s'ajouter les éléments métaphoriques, visuels ou même "pittoresques» (par exemple, syndrome du bâtiment malsain) qui peuvent attirer davantage l'attention du sujet lors de la sélection de la variante la plus familière, ainsi que des éléments d'ordre pragmatique tels que la fréquence d'usage du terme dans les textes de vulgarisation. Il découle donc de nos résultats que les termes les plus fréquents au sein des corpus électroniques sont les plus familiers.

Enfin, le texte est constitué de traits associés à la situation communicative qui influencent la perception des termes d'un texte considérés comme familiers. On peut citer à ce propos certains de ces traits essentiels: le degré de spécialisation d'un texte (vulgarisation, semi-spécialisé ou spécialisé), texte oral ou écrit (ou bien écrit pour être lu à voix haute, texte oral transcrit, etc.), le niveau de difficulté du texte (selon le genre textuel, la densité terminologique, le sujet et domaine de spécialité, le type et le nombre de ressources documentaires disponibles, la commande de traduction, le format du texte, les outils de traduction indispensables, etc.) ou le rapport entre le texte, le contexte et les images et illustrations à l'appui. Notre expérience empirique nous a permis de confirmer que le terme qualifié comme plus familier est généralement le moins spécialisé. Cela est sans doute dû au fait que de nos jours il y a de plus en plus de sites Web de vulgarisation portant sur des sujets médicaux qui prétendent diffuser des connaissances générales de manière accessible. Par conséquent, nous considérons que des éléments communicatifs et situationnels tels que le contexte et les images peuvent favoriser la perception d'un terme en tant que familier.

\section{Conclusion}

En définitive, dans cet article, l'accent a été mis sur la portée des bases de données fournissant des inventaires de variantes terminologiques et l'importance que revêt le fait de comparer les éléments d'une base de données telle que VariMed avec les intuitions lexicales des usagers issues de leurs expériences. Nous avons notamment analysé les enjeux du phénomène de familiarité et ses rapports avec les choix du traducteur. La présente étude a mis en évidence ces relations, et notamment les tendances suivantes:

Les termes désignant les maladies les plus courantes sont souvent les plus familiers; le lecteur du texte cible les comprendra sans doute facilement, d'où une moindre incertitude pour le traducteur au moment de les traduire. Il en va de même pour les termes présentant une morphologie similaire dans la langue d'arrivée: leur emploi dans le texte cible garantit une équivalence appropriée même quand le terme est analogue (Tercedor-Sánchez 2011; Tercedor-Sánchez, López-Rodríguez et AlarcónNavío 2013), ce qui permet, là encore, de réduire l'incertitude.

Toutefois, les termes spécialisés à racine gréco-latine font exception car généralement les sujets de l'expérience menée ne les ont pas reconnus en tant que termes familiers. Si le traducteur décide de les employer, il serait souhaitable par conséquent qu'il ait recours à une paraphrase ou à une explicitation jointe au terme; l'utilisation des stratégies de traduction s'avère bien être le meilleur moyen de réduire l'incertitude en traduction. 
Ces observations conduisent à établir une corrélation entre le nombre de choix lexicaux pour un concept et le degré d'incertitude dans la détermination du terme le plus familier, et donc dans la détermination de l'équivalent dans le texte cible.

Le choix d'un terme hyperonyme ou d'un niveau de spécialisation intermédiaire favorise la reconnaissance du caractère familier de celui-ci, ce qui réduit d'autant les risques d'incompréhension de la part du lecteur et permet d'atteindre un degré satisfaisant de certitude quant au choix le plus adapté à une communication réussie. Enfin, notre expérience a permis de montrer de façon empirique l'impact de la langue maternelle du traducteur sur la reconnaissance des termes de la langue cible comme étant familiers. Des phénomènes d'interférence sont également intervenus, élargissant ainsi les possibilités de choix de traduction - certains d'entre eux d'ailleurs se révélant parfois faux -, ce qui a mis en évidence la complexité de l'activité traduisante en tant que processus de prise de décision et de sélection parmi les différents choix.

Les traducteurs professionnels et les traducteurs en formation doivent souvent faire face à des choix de variantes spécialisées. Il convient donc de créer des outils, des exercices et des tâches de traduction destinés à mettre en évidence la richesse de la terminologie médicale permettant aux traducteurs d'améliorer leurs performances traductionnelles.

En guise de conclusion, nous pouvons affirmer que la familiarité fait partie des motivations qui dictent la variation dénominative, et qu'elle est bien différente de la connaissance exacte infaillible des termes et des concepts afférents. L'exploration de la familiarité et la subjectivité qu'elle comporte deviennent alors l'un des enjeux de l'activité traduisante. Cette quête est faite de choix et d'incertitude, suivie de la validation ou non de la prise de décision. C'est là matière à réflexion et à action pour les étudiants de traduction souhaitant maîtriser les subtilités du langage médical. Il est bien connu que le doute est formateur, mais la présence de l'incertitude et sa gestion adéquate constituent sans doute la force secrète de la traduction.

\section{NOTES}

* Étude menée dans le cadre du projet «VariMed: Variación denominativa en medicina: recurso multimodal multilingüe para investigación y divulgación (FFI2011-23120)» [VariMed: Variation dénominative en médecine. Ressource multifonctionnelle plurilingue pour la recherche et la vulgarisation] (http://varimed.ugr.es), du projet «CombiMed: Léxico combinatorio en medicina: cognición, texto y contexto (FFI2014-51899R) [CombiMed: Lexique combinatoire en médecine: cognition, texte et contexte] et du projet d'innovation didactique «Recursos audiovisuales para el fomento de la salud en Europa: subtitulado accesible y traducción (14-39)» [Ressources audiovisuelles pour le développement de la santé en Europe: sous-titrage accessible et traduction] (https:// tradusaluda.wordpress.com) du vice-rectorat du corps enseignant et d'ordonnancement académique de l'Université de Grenade. VariMed est un projet de recherche et d'innovation financé par le ministère de l'Innovation et de la Science d'Espagne, avec la participation des chercheurs du groupe Lexicon (http://lexicon.ugr.es) et des universités suivantes: Université de Grenade, Université Pablo de Olavide de Séville, Université de Castille-la Manche, Université de Valladolid, Université Rutgers (New Jersey) et Université Carleton (Ottawa). Les mêmes chercheurs forment l'équipe de CombiMed, projet financé par le Ministère de l'Économie et de la Compétitivité d'Espagne.

1. Le corpus EsTenTen11 (European, Tree Tagger) a été consulté à l'aide du système de recherche de corpus Sketch Engine (https://the.sketchengine.co.uk). Le corpus EsTenTen11 (Eu) contient plus de 2 milliards de mots. 


\section{RÉFÉRENCES}

Aijón, Miguel Ángel et Serrano, María José (2010): Las bases cognitivas del estilo lingüístico. Sociolinguistic studies. 4(1):115-144.

Alderson, J. Charles (2007): Judging the frequency of English words. Applied Linguistics. 28(3):383-409.

Atkinson, Richard C. et JuOLA, James F. (1973): Factors influencing speed and accuracy of word recognition. In: Sylvan Kornblum, dir. Fourth international symposium on attention and performance. New York: Academic Press, 583-611.

BAKer, Mona (1992): In Other Words: A coursebook on translation. Londres/New York: Routledge.

BARSALOU, Lawrence W. (2003): Situated simulation in the human conceptual system. Language and Cognitive Process. 18:513-562.

Bowker, Lynne et Hawkins, Shane (2006). Variation in the Organization of Medical Terms: Exploring Some Motivations for Term Choice. Terminology. 12(1):79-110.

Bromme, Rainer, RAмвоw, Riklef et Nückles, Matthias (2001): Expertise and estimating what other people know : the influence of professional experience and type of knowledge. Journal of Experimental Psychology: Applied. 7(4):317-330.

CABré, Maria Teresa (1999): Terminology Theory, Methods and Applications. Amsterdam/Philadelphie: John Benjamins.

Connell, Louise, Lynott, Dermot et Dreyer, Felix (2012): A functional role for modalityspecific perceptual systems in conceptual representations. PLoS One. 7(3):e33321.

Connine, Cynthia M., Mullennix, John, Shernoff, Eve et Yelen, Jennifer (1990): Word familiarity and frequency in visual and auditory word recognition. Journal of Experimental Psychology: Learning, Memory, and Cognition. 16(6):1084-1096.

Delgado Corujo, María Isabel (1988): Estudios normativos sobre parámetros de familiaridad subjetiva, concreción e imaginabilidad en textos narrativos y expositivos. Mémoire de licence non publié. San Cristóbal de La Laguna: Universidad de La Laguna.

Fernández Silva, Sabela (2010): Variación terminológica y cognición. Factores cognitivos en la denominación del concepto especializado. Thèse de doctorat non publiée. Barcelone: Universitat Pompeu Fabra.

FreiXA, Judith (2006): Causes of denominative variation in terminology: A typology proposal. Terminology. 12(1):51-77.

Glanzer, Murray et Adams, John K. (1985): The mirror effect in recognition memory. Memory and Cognition. 13(1):8-20.

Gómez Veiga, Isabel, Carriedo López, Nuria, Rucián Gallego, Mercedes et Vila Cháves, José Óscar (2010): Estudio normativo de ambigüedad léxica en castellano en niños y en adultos. Psicológica. 31:25-47.

GreGG, Vernon (1976): Word frequency, recognition, and recall. In: John Brown, dir. Recall and recognition. New York: Wiley, 183-216.

GuZmán Rosquete, Remedios et Jiménez GonZÁlez, Juan Eugenio (2001) : Estudio normativo sobre parámetros psicolingüísticos en niños de 6 a 8 años: la familiaridad subjetiva. Cognitiva. 13(2):153-191.

Halliday, Michael Alexander Kirkwood, McIntosh, Angus et Strevens, Peter (1964): The Linguistic Sciences and Language Teaching. Londres: Longman.

Hatim, Basil et Mason, Ian (1990): Discourse and the Translator. New York: Longman.

JACOBY, Larry L. (1991): A process dissociation framework: Separating automatic from intentional uses of memory. Journal of Memory and Language. 30(5):513-541.

JACOBY, Larry L. et Kelley, Clarence M. (1992): Unconscious influences of memory: Dissociations and automaticity. In: A. David Milner et Michael D. RugG, dir. The Neuropsychology of Consciousness. San Diego: Academic Press, 201-233.

Jacoby, Larry L., Yonelinas, Andrew P. et Jennings, Janine M. (1997): The relation between conscious and unconscious (automatic) influences: A declaration of independence. In: 
Jonathan D. Cohen et Jonathan W. Schooler, dir. Scientific approaches to consciousness. Hillsdale, NJ: Erlbaum, 13-47.

Kuperman, Victor et VAn Dyke, Julie A. (2013) : Reassessing word frequency as a determinant of word recognition for skilled and unskilled readers. Journal of Experimental Psychology: Human Perception and Performance. 39(3):802-823.

LAKoff, George (1987): Women, Fire, and Dangerous Things: What Categories Reveal about the Mind. Chicago: University of Chicago.

MAndler, George (1980): Recognizing: The judgment of previous occurrence. Psychological Review. 87(3):252-271

Navarro, Fernando (2009): La precisión del lenguaje en la traducción médica. La redacción médica como profesión. Qué es y qué hace el redactor de textos médicos. Numéro spécial. Quaderns de la Fundació Dr. Antoni Esteve. 17:89-104.

Nord, Christiane (2005): Text Analysis in Translation: Theory, Methodology, and Didactic Application of a Model for Translation-oriented Text Analysis. Amsterdam/New York: Rodopi.

Prieto-Velasco, Juan Antonio et Tercedor-Sánchez, Maribel (2014): The embodied nature of medical concepts: image schemas and language for PAIN. Cognitive Processing. 15(3):283296.

Rogers, Margaret (2004): Multidimensionality in concept systems: A bilingual textual perspective. Terminology. 10(2):215-240.

Rosch, Eleanor (1978): Principles of Categorization. In: Eleanor Rosch et Barbara B. Lloyd, dir. Cognition and Categorization. Hillsdale, NJ: Lawrence Erlbaum Associates, 27-48.

Saldanha, Gabriela et O'Brien, Sharon (2014): Research Methodologies in Translation Studies. Londres: Routledge.

Tercedor-Sánchez, Maribel (2002): Descripción y representación de la variación terminológica: el caso de la dimensión tipos de cáncer. In: Pamela Faber et Catalina Jiménez, dir. Investigar en Terminología. Grenade: Comares, 199-214.

Tercedor-Sánchez, Maribel (2011): The cognitive dynamics of terminological variation. Terminology. 17(2):181-197.

Tercedor-SÁnchez, Maribel et López-Rodríguez, Clara Inés (2012): Access to health in an intercultural setting: the role of corpora and images in grasping term variation. Translation and knowledge mediation in medical and health settings. Numéro spécial. Linguistica Antverpiensia New Series - Themes in Translation Studies. 11:247-268.

Tercedor-SÁnchez, Maribel, López-Rodríguez, Clara Inés et Alarcón-Navío, Esperanza (2013): Identifying translation features in multiword lexical units. Belgian Journal of Linguistics. 27:87-109.

Tercedor-Sánchez, Maribel, López-Rodríguez, Clara Inés et Prieto-Velasco, Juan Antonio (2014): También los pacientes hacen terminología: retos del proyecto VariMed.Panace@, Rev. de Medicina, Lenguaje y Traducción. 15(39):95-102.

Tercedor-Sánchez, Maribel et Méndez Cendón, Beatriz (2000): Fraseología y variación terminológica: estudio descriptivo en corpora biomédicos. Terminologie et Traduction. 2:82-100.

Wierzbicka, Anna (1995): Universal Semantic Primitives as a Basis for Lexical Semantics. Folia Lingüística. 30(1-2):149-169.

Yap, Melvin J., Balota, David A., Sibley, Daragh E. et Ratcliff, Roger (2012): Individual differences in visual word recognition: Insights from the English lexicon project. Journal of Experimental Psychology: Human Perception and Performance. 38(1):53-79.

Yonelinas, Andrew P. (2002): The Nature of Recollection and Familiarity: A Review of 30 Years of Research. Journal of Memory and Language. 46:441-517. 
ANNEXE

Les 30 stimuli pour l'étude de la familiarité: définitions et variantes dénominatives

\begin{tabular}{|c|c|c|}
\hline $\begin{array}{l}\text { Légende } \\
\text { Caractères gras: définition fournie aux sujets en tant que stimulus. } \\
\text { PETITES MAJUSCULES EN CARACTÈRES GRAS: variante désignant le concept } \\
\text { dans VariMed. } \\
\text { ^ Terme distracteur }\end{array}$ & & \\
\hline 1. Infección por cándida localizada en la vagina. & ES L1 & FR L1 \\
\hline CANDIDIASIS VAGINAL & 40 & 4 \\
\hline Candidiasis vulvo-vaginal & 0 & 2 \\
\hline *Infección vaginal & 2 & 30 \\
\hline Infección micótica vaginal & 1 & 12 \\
\hline Infección vaginal por hongos & 5 & 1 \\
\hline NS/NC & 2 & 1 \\
\hline $\begin{array}{l}\text { 2. Inflamación de una o varias amígdalas linfáticas. La etiología de la } \\
\text { amigdalitis palatina aguda se asemeja a la de la faringitis. Se manifiesta } \\
\text { clínicamente por dolor local intenso, adenopatías cervicales, disminución de la } \\
\text { movilidad del velo del paladar, voz gangosa, alteraciones para la deglución y, } \\
\text { en algunos casos, apnea. }\end{array}$ & ES L1 & FR L1 \\
\hline AMIGDALITIS & 26 & 16 \\
\hline${ }^{\star}$ Anginoides & 1 & 0 \\
\hline Tonsilitis & 0 & 6 \\
\hline Anginas & 23 & 28 \\
\hline NS/NC & 0 & 0 \\
\hline $\begin{array}{l}\text { 3. Afección cerebrovascular súbita producida por una falta de riego sanguíneo } \\
\text { en una zona del cerebro, debida a la rotura de un vaso sanguíneo o a un } \\
\text { coágulo. Se caracteriza por una pérdida súbita y sin convulsiones de la función } \\
\text { neurológica. }\end{array}$ & ES L1 & FR L1 \\
\hline Accidente cerebral vascular & 1 & 2 \\
\hline Accidente cerebrovascular & 2 & 16 \\
\hline ACV & 0 & 28 \\
\hline Apoplejía & 0 & 0 \\
\hline Derrame cerebral & 34 & 0 \\
\hline ICTUS & 13 & 0 \\
\hline${ }^{*}$ Accidente cerebral & 0 & 4 \\
\hline $\mathrm{NS} / \mathrm{NC}$ & 0 & 0 \\
\hline $\begin{array}{l}\text { 4. Afección cutánea caracterizada por un taponamiento de las glándulas } \\
\text { sebáceas o los folículos pilosos que puede provocar inflamación e infección. }\end{array}$ & ES L1 & FR L1 \\
\hline Granos & 13 & 10 \\
\hline Espinillas & 6 & 4 \\
\hline Granitos & 0 & 4 \\
\hline Barros & 1 & 0 \\
\hline ACNÉ & 30 & 31 \\
\hline${ }^{*}$ Pupas & 0 & 1 \\
\hline NS/NC & 0 & 0 \\
\hline
\end{tabular}




\begin{tabular}{|c|c|c|}
\hline 5. Evacuación de heces líquidas más de tres veces al día. & ES L1 & FR L1 \\
\hline Colitis & 4 & 2 \\
\hline${ }^{\star}$ Cólico & 6 & 4 \\
\hline Descomposición & 1 & 0 \\
\hline DiARREA & 38 & 43 \\
\hline Cagalera & 1 & 0 \\
\hline $\mathrm{NS} / \mathrm{NC}$ & 0 & 1 \\
\hline $\begin{array}{l}\text { 6. Infección respiratoria causada por virus que es enormemente contagiosa y } \\
\text { virulenta especialmente en niños y ancianos. }\end{array}$ & ES L1 & FR L1 \\
\hline${ }^{*}$ Gripe vírica & 7 & 21 \\
\hline Influenza & 0 & 2 \\
\hline GRIPE & 43 & 26 \\
\hline $\mathrm{NS} / \mathrm{NC}$ & 0 & 1 \\
\hline $\begin{array}{l}\text { 7. Proceso infeccioso de las vías respiratorias altas, esencialmente de las fosas } \\
\text { nasales, de breve duración (de } 5 \text { a } 7 \text { días), y de naturaleza benigna, muy común } \\
\text { en los meses de invierno en la población general. Es muy contagioso. }\end{array}$ & ES L1 & FR L1 \\
\hline Constipado & 1 & 10 \\
\hline${ }^{\star}$ Costipado & 1 & 0 \\
\hline Enfriamiento & 0 & 8 \\
\hline Resfrío & 0 & 22 \\
\hline RESFRIADO & 36 & 2 \\
\hline Catarro & 5 & 3 \\
\hline Catarro común & 3 & 0 \\
\hline Resfriado común & 4 & 4 \\
\hline NS/NC & 0 & 1 \\
\hline $\begin{array}{l}\text { 8. Enfermedad contagiosa exantemática causada por el virus de la varicela- } \\
\text { zóster (VVZ) que afecta habitualmente a los niños. Su signo más característico } \\
\text { es una erupción en la piel que aparece en forma de pequeños granos que en } \\
\text { poco tiempo se convierten en vesículas (ampollas llenas de líquido) que pican. }\end{array}$ & ES L1 & FR L1 \\
\hline VARICELA & 37 & 34 \\
\hline Payuelas & 6 & 1 \\
\hline${ }^{\star P}$ Picota & 0 & 8 \\
\hline Herpes zóster & 7 & 7 \\
\hline $\mathrm{NS} / \mathrm{NC}$ & 0 & 0 \\
\hline $\begin{array}{l}\text { 9. Enfermedad infecciosa contagiosa que afecta habitualmente a los niños, } \\
\text { causada por un paramixovirus y localizada fundamentalmente en una o ambas } \\
\text { glándulas parótidas. }\end{array}$ & ES L1 & FR L1 \\
\hline Paperas & 41 & 8 \\
\hline Parotiditis & 6 & 23 \\
\hline *Paramixotis & 2 & 11 \\
\hline Parotiditis epidémica & 1 & 2 \\
\hline NS/NC & 0 & 6 \\
\hline $\begin{array}{l}\text { 10. Conjuntivitis causada por la infección de ciertas bacterias en el ojo. Se } \\
\text { caracteriza por intensa inflamación conjuntival y secreción purulenta } \\
\text { abundante. }\end{array}$ & ES L1 & FR L1 \\
\hline Conjuntivitis purulenta & 4 & 7 \\
\hline${ }^{\star}$ Conjuntivitis inflamatoria & 13 & 16 \\
\hline CONJUNTIVITIS BACTERIANA & 33 & 26 \\
\hline $\mathrm{NS} / \mathrm{NC}$ & 0 & 1 \\
\hline
\end{tabular}




\begin{tabular}{|c|c|c|}
\hline $\begin{array}{l}\text { 11. Forma rara de conjuntivitis crónica caracterizada por la formación } \\
\text { recurrente de lesiones pseudomembranosas, generalmente a nivel de la } \\
\text { superficie palpebral. }\end{array}$ & ES L1 & FR L1 \\
\hline Conjuntivitis lignea & 6 & 0 \\
\hline${ }^{\star}$ Conjuntivitis parpebral & 22 & 36 \\
\hline CONJUNTIVITIS LEÑOSA & 21 & 10 \\
\hline $\mathrm{NS} / \mathrm{NC}$ & 1 & 4 \\
\hline $\begin{array}{l}\text { 12. Disminución en la frecuencia de la defecación o en el tamaño y consistencia } \\
\text { de las heces. }\end{array}$ & ES L1 & FR L1 \\
\hline ESTREÑIMIENTO & 49 & 15 \\
\hline Constipación & 1 & 35 \\
\hline${ }^{*}$ Atranque & 0 & 0 \\
\hline $\mathrm{NS} / \mathrm{NC}$ & 0 & 0 \\
\hline $\begin{array}{l}\text { 13. Tipo de alergia estacional que se produce en primavera causada por el } \\
\text { polen. }\end{array}$ & ES L1 & FR L1 \\
\hline ALERGIA PRIMAVERAL & 48 & 35 \\
\hline Fiebre del heno & 2 & 11 \\
\hline${ }^{\star}$ Alergitis & 0 & 4 \\
\hline NS/NC & 0 & 0 \\
\hline $\begin{array}{l}\text { 14. Enfermedad inflamatoria que aparece como secuela de una infección por } \\
\text { estreptococo beta-hemolítico Grupo A (Streptococcus pyogenes), habitualmente } \\
\text { faringoamigdalitis o escarlatina. Afecta el corazón, articulaciones, sistema } \\
\text { nervioso central y tejido subcutáneo. }\end{array}$ & ES L1 & FR L1 \\
\hline Artritis reumática aguda & 12 & 10 \\
\hline Fiebre REUMÁtica & 5 & 8 \\
\hline Fiebre reumática aguda & 0 & 4 \\
\hline FR & 0 & 0 \\
\hline${ }^{*}$ Artrosis & 14 & 4 \\
\hline Reumatismo articular agudo & 3 & 2 \\
\hline Reuma & 8 & 2 \\
\hline Reumatismo & 3 & 16 \\
\hline Reúma & 5 & 2 \\
\hline $\mathrm{NS} / \mathrm{NC}$ & 0 & 2 \\
\hline $\begin{array}{l}\text { 15. Dolor de cabeza que puede estar causado por múltiples factores, aunque el } \\
\text { más frecuente es la tensión. }\end{array}$ & ES L1 & FR L1 \\
\hline Dolor de cabeza & 11 & 4 \\
\hline *Migraña & 24 & 36 \\
\hline Cefalea & 15 & 10 \\
\hline $\mathrm{NS} / \mathrm{NC}$ & 0 & 0 \\
\hline $\begin{array}{l}\text { 16. Percepción de un sonido continuo o intermitente que puede ocurrir en un } \\
\text { oído o en ambos. }\end{array}$ & ES L1 & FR L1 \\
\hline Zumbido & 13 & 2 \\
\hline Pitido & 33 & 2 \\
\hline Tinnitus & 0 & 2 \\
\hline ACúfENo & 2 & 37 \\
\hline${ }^{*}$ Acufenitis & 2 & 2 \\
\hline Tinnitus aurium & 0 & 2 \\
\hline $\mathrm{NS} / \mathrm{NC}$ & 0 & 3 \\
\hline
\end{tabular}




\begin{tabular}{|c|c|c|}
\hline 17. Pérdida completa de la capacidad auditiva. & ES L1 & FR L1 \\
\hline Sordera total & 25 & 25 \\
\hline Sordera completa & 5 & 17 \\
\hline${ }^{\star}$ Sordera parcial & 0 & 0 \\
\hline Sordera & 16 & 8 \\
\hline ANACUSIa & 4 & 0 \\
\hline $\mathrm{NS} / \mathrm{NC}$ & 0 & 0 \\
\hline 18. Lesión de la membrana mucosa que recubre las paredes del estómago. & ES L1 & FR L1 \\
\hline Úlcera & 10 & 10 \\
\hline ÚLCERA GÁSTRICA & 18 & 28 \\
\hline *Úlcera gastrosa & 0 & 2 \\
\hline Úlcera de estómago & 13 & 8 \\
\hline Úlcera estomacal & 9 & 2 \\
\hline $\mathrm{NS} / \mathrm{NC}$ & 0 & 0 \\
\hline $\begin{array}{l}\text { 19. Cualquier neoplasia maligna que se forma en los tejidos del pulmón, por lo } \\
\text { general, en células que recubren las vías respiratorias. Los dos tipos más } \\
\text { importantes son el cáncer de pulmón de células pequeñas y el cáncer de } \\
\text { pulmón de células no pequeñas. El tabaco es la principal causa de este cáncer. }\end{array}$ & ES L1 & FR L1 \\
\hline Cáncer pulmonar & 9 & 10 \\
\hline CÁNCER dE PULMÓN & 41 & 15 \\
\hline${ }^{\star}$ Cáncer de pulmones & 0 & 17 \\
\hline Cáncer broncopulmonar & 0 & 8 \\
\hline $\mathrm{NS} / \mathrm{NC}$ & 0 & 0 \\
\hline $\begin{array}{l}\text { 20. Cáncer que se forma en los tejidos de la mama, por lo general, en los } \\
\text { conductos galactóforos (tubos que llevan leche al pezón) y los lobulillos } \\
\text { (glándulas que producen leche). Se puede presentar tanto en hombres como en } \\
\text { mujeres, aunque en mujeres es más frecuente. Va acompañado de una masa no } \\
\text { dolorosa, y puede causar hinchazón del seno, hendiduras en la piel, dolor en el } \\
\text { seno, retracción del pezón y secreción anormal del pezón. }\end{array}$ & ES L1 & FR L1 \\
\hline CÁNCER DE MAMA & 49 & 25 \\
\hline Cáncer de seno & 0 & 4 \\
\hline *Cáncer de la mama & 0 & 9 \\
\hline Cáncer mamario & 0 & 12 \\
\hline Neoplasia de la mama & 0 & 0 \\
\hline Tumor mamario & 1 & 0 \\
\hline Tumor de la mama & 0 & 0 \\
\hline $\mathrm{NS} / \mathrm{NC}$ & 0 & 0 \\
\hline $\begin{array}{l}\text { 21. Tumor maligno localizado en el esófago, cuyos síntomas incluyen } \\
\text { dificultad para tragar líquidos y sólidos (disfagia), presión y quemazón de } \\
\text { garganta. Sus tipos son carcinoma epidermoide y adenocarcinoma. Una de sus } \\
\text { principales causas es el tabaco. }\end{array}$ & ES L1 & FR L1 \\
\hline CÁNCER DE ESÓFAGO & 50 & 37 \\
\hline Cáncer esofágico & 0 & 2 \\
\hline Neoplasia maligna de esófago & 0 & 0 \\
\hline Neoplasia esofágica & 0 & 4 \\
\hline${ }^{\star}$ Cáncer de la epidermis & 0 & 0 \\
\hline Tumor maligno de esófago & 0 & 7 \\
\hline $\mathrm{NS} / \mathrm{NC}$ & 0 & 0 \\
\hline
\end{tabular}




\begin{tabular}{|c|c|c|}
\hline 22. Pérdida anormal o rarefacción del pelo. & ES L1 & FR L1 \\
\hline Alopecia & 37 & 5 \\
\hline Calvicie & 12 & 43 \\
\hline${ }^{*}$ Calvas & 1 & 2 \\
\hline $\mathrm{NS} / \mathrm{NC}$ & 0 & 0 \\
\hline $\begin{array}{l}\text { 23. Venas superficiales, dolorosas, retorcidas y agrandadas que resultan del } \\
\text { funcionamiento deficiente de las válvulas y que suelen producirse } \\
\text { principalmente en las venas de las piernas, aunque se pueden presentar en } \\
\text { otras partes. }\end{array}$ & ES L1 & FR L1 \\
\hline Venas varicosas & 4 & 3 \\
\hline Arañas vasculares & 1 & 3 \\
\hline VARICES & 42 & 36 \\
\hline Varices de piernas & 1 & 2 \\
\hline${ }^{\star}$ Evarices & 1 & 4 \\
\hline $\mathrm{NS} / \mathrm{NC}$ & 1 & 2 \\
\hline $\begin{array}{l}\text { 24. Deformidad de los huesos que unen el dedo gordo del pie y que produce un } \\
\text { abultamiento. }\end{array}$ & ES L1 & FR L1 \\
\hline Juanete & 45 & 10 \\
\hline HaLLUX VALGUS & 1 & 15 \\
\hline${ }^{\star}$ Hallux del valgo & 2 & 20 \\
\hline $\mathrm{NS} / \mathrm{NC}$ & 2 & 5 \\
\hline 25. Mordisquear, desgastar o comer las uñas con los dientes de forma habitual. & ES L1 & FR L1 \\
\hline Morderse las uñas & 32 & 9 \\
\hline ONICOFAgIa & 5 & 16 \\
\hline Comerse las uñas & 12 & 24 \\
\hline${ }^{*}$ Fagitosis & 1 & 0 \\
\hline NS/NC & 0 & 1 \\
\hline 26. Pérdida anormal del apetito. & ES L1 & FR L1 \\
\hline Falta de apetito & 33 & 37 \\
\hline Inapetencia & 13 & 5 \\
\hline ANOREXIA & 4 & 8 \\
\hline${ }^{\star B}$ Bulimia & 0 & 0 \\
\hline $\mathrm{NS} / \mathrm{NC}$ & 0 & 0 \\
\hline $\begin{array}{l}\text { 27. Sensación de necesidad de rascarse la piel o mucosas, normalmente como } \\
\text { resultado de una irritación. }\end{array}$ & ES L1 & FR L1 \\
\hline Comezón & 1 & 4 \\
\hline Picor & 24 & 6 \\
\hline Picazón & 5 & 8 \\
\hline *Irritación & 16 & 26 \\
\hline PruRito & 4 & 4 \\
\hline NS/NC & 0 & 2 \\
\hline 28. Olor fétido que se emite al respirar o abrir la boca. & ES L1 & FR L1 \\
\hline *Fetidez & 0 & 4 \\
\hline HALITOSIS & 34 & 11 \\
\hline Mal aliento & 16 & 35 \\
\hline $\mathrm{NS} / \mathrm{NC}$ & 0 & 0 \\
\hline
\end{tabular}




\begin{tabular}{|l|c|c|}
\hline $\begin{array}{l}\text { 29. Desviación espontánea y permanente de los ejes oculares. Puede ser } \\
\text { unilateral o bilateral. }\end{array}$ & ES L1 & FR L1 \\
\hline EsTRABISMo & 32 & 48 \\
\hline Bizquera & 17 & 0 \\
\hline${ }^{*}$ Ojo tuerto & 1 & 0 \\
\hline NS/NC & 0 & 2 \\
\hline $\begin{array}{l}\text { 30. Error de refracción en el que los rayos procedentes del infinito convergen } \\
\text { en un punto posterior al plano de la retina cuando el ojo se encuentra en } \\
\text { situación de reposo, lo que provoca una mala visión de cerca. }\end{array}$ & ES L1 & FR L1 \\
\hline${ }^{*}$ Metropía & 2 & 7 \\
\hline Hiperpresbiopía & 1 & 6 \\
\hline Hiperopía & 0 & 3 \\
\hline Hiperopsia & 1 & 2 \\
\hline HIPERMETROPÍA & 46 & 29 \\
\hline NS/NC & 0 & 3 \\
\hline
\end{tabular}

